
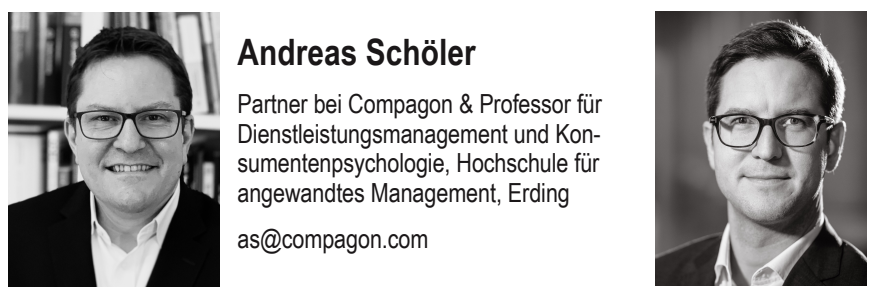

Dominik van Aaken

Partner bei Compagon \& Professor für strategisches Management und Organisation an der Universität Salzburg

dva@compagon.com

\title{
Service Design und Failing Forward
}

\author{
Von der Gestaltung von Kundenreisen und der Möglichkeit, aus dem Scheitern \\ zu lernen
}

Service Design hilft Kundenerlebnisse wirksam zu gestalten. In diesem Artikel zeigen wir die zusätzlichen Herausforderungen auf, die bei der Gestaltung von Kundenreisen in einer digitalen Welt zu beachten sind. Aufgrund der vielfältigen Herausforderungen, können entsprechende Initiativen leicht scheitern. Wir erläutern die Grundvoraussetzungen, um aus diesem Scheitern im Sinne eines Failing Forward zu lernen.

\section{Service Design in einer digitalisierten Welt}

Service Design beschreibt die Gestaltung von Dienstleistungen aus Nutzerperspektive. ${ }^{1}$ Im Fokus steht dabei das Design der tangiblen (greifbaren) und intangiblen Dienstleistungselemente, die Kunden unmittelbar erleben. Ein Kernkonzept des Service Design ist der Ansatz der sog. „Kundenreise“. Es stellt den relevanten Erlebensprozess von Kunden rund um die Nutzung einer Dienstleistung in einer visualisierten Form dar.

Bei einer Kundenreise werden vor allem die Kontaktpunkte zwischen (verschiedenen, aber typischen) KundInnen und einem Dienstleister vom „Anfang“ bis „Ende“ (also beispielsweise von dem Aufkommen eines Serviceanliegens bis zur Lösung aus Kundensicht) dargestellt und analysiert. Grundüberzeugung ist, dass der unmittelbare (persönliche, mediale oder digitale) Kundenkontakt bei Dienstleistungen zentral für die wahrgenommene Qualität der Dienstleistungsprozesse ist. Kundenreisen können weiter ergänzt werden. Neben den unmittelbaren Kontakten zwischen KundInnen und Unternehmen können zu einer Kundenreise auch die Tätigkeiten abgebildet werden, die für KundInnen verdeckt bleiben. Dabei handelt es sich um alle anbieterseitigen Aufgaben, die für die Erbringung einer Leistung erforderlich sind. Dies entspricht dem Ansatz 
des „Service Blueprinting“. Kundenerlebnispunkte werden hier von internen und damit unsichtbaren Tätigkeiten durch eine "Line-of-Visibility" getrennt. In der Literatur hat sich zur Differenzierung auch die Theatermetapher „Frontstage“ für Kundenkontaktpunkte und „Backstage“ für interne Tätigkeiten etabliert. ${ }^{2}$ Die Konzepte der Kundenreise und des Service Blueprintings lassen sich natürlich auch verbinden. Abbildung 1 zeigt dies beispielhaft für den Kauf einer Waschmaschine in einem Elektronikfachmarkt.

Das Design von Kundenreisen gehört mittlerweile zum klassischen Methodenarsenal des Dienstleistungsmanagements. In den letzten Jahren wird das Design von Kundenprozessen allerdings komplexer, da man eine immer weiter verbreitete Verbindung von analogen und digitalisierten Dienstleistungsprozessen beobachten kann. Heute starten bspw. Serviceprozesse auf der Webseite eines Händlers, führen zu einem Beratungsgespräch via Video oder Chat, münden in eine Online-Bestellung sowie in ein persönliches Abholen vor Ort in der Filiale als Click \& Meet. Oder anders herum: KundInnen informieren sich in einer Filiale, bestellen dann über eine Website und lassen sich das Produkt nach Hause liefern. Digitale und analoge Leistungselemente lassen sich beinah beliebig kombinieren. Wie auch immer die konkrete Kombination der digitalen und analogen Leistungselemente aussieht, die Inanspruchnahme mehrerer Kanäle (analog und digital) und insbesondere auch der Wechsel zwischen unterschiedlichen Kanälen stellt das Design von Kundenprozessen vor neuen Herausforderungen. Diese wollen wir hier vorstellen.

\section{Gestaltung von Kanalübergängen entlang von Kundenreisen}

Das Design des Kundenerlebnisses muss nicht nur in den einzelnen Kanälen hervorragend sein, sondern auch an den Übergängen. Dies erfordert, dass das Gesamtbild von möglichen Kundenreisen aus Kundensicht im Unternehmen selbst nachvollzogen werden muss. Konkret bedeutet dies beispielsweise, dass in einer Filiale auch auf die Suchund Kaufhistorie von KundInnen aus dem Online-Shopping zurückgegriffen werden kann, so dass Mitarbeitende vor Ort direkt darauf aufbauen können. Das gleiche gilt für eine telefonische Beratung, bei der Mitarbeitende direkt in die Online-Session von KundInnen einsteigen können. Kanalübergänge konsistent zu gestalten bedeutet auch, Kundlnnen bewusst und bedarfsgerecht von einem Kanal in den anderen Kanal überzuleiten. Die Media-Richness-Theorie ${ }^{3}$ gibt es an die Hand: Der Kanal muss zum Bedarf des Kunden passen. In einem Online-Chat über eine Immobilienfinanzierung zu sprechen ist möglicherweise nicht zielführend. Hier sind Überleitungsprozesse in den passenden Kanal gefragt. Bei der Entwicklung von Kundenreisen müssen also Kanalübergänge aus Kundensicht betrachtet werden. Es muss geklärt werden, welche Übergänge zwischen Kanälen wahrscheinlich sind, was an diesen Übergängen passieren sollte, damit Kundlnnen diese als reibungslos wahrnehmen und wo "Gefahren“ bzw. Unzufriedenheitstreiber liegen. 


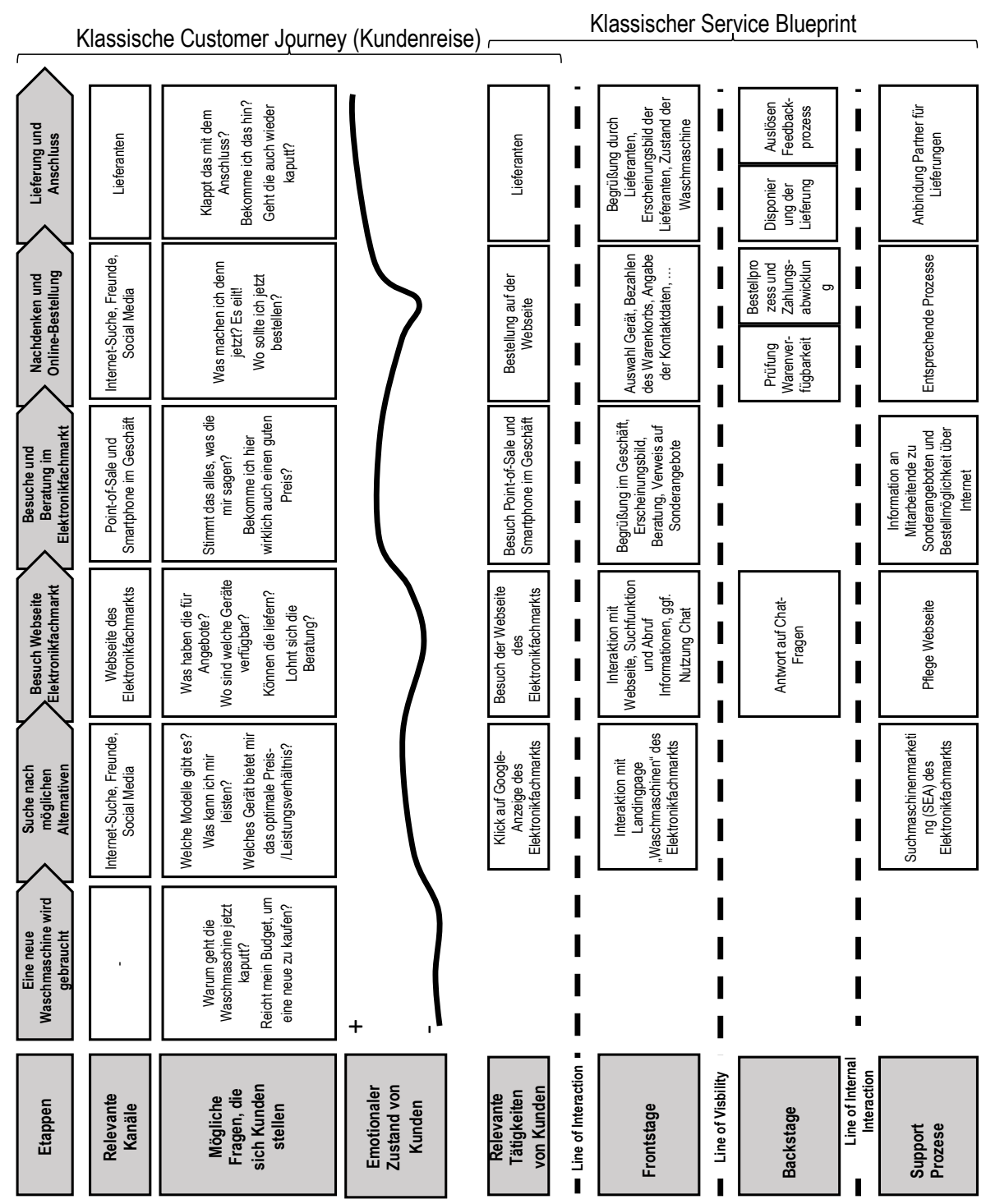

Abbildung 1: Exemplarische Kundenreise eines Waschmaschinenkaufs bei einem Elektronikfachmarkt 


\section{Konsistente Gesamterlebnisse in allen Kanälen}

Selbstverständlich muss das Kundenerlebnis im Gesamtprozess stimmig und konsistent sein. Dies bedeutet, dass das Teilerleben von KundInnen in den unterschiedlichen Kanälen ein passendes und in sich stimmiges Gesamtbild abgeben muss. Die Abstimmung über die unterschiedlichen Kanäle und Kontaktpunkte kann hier komplexe Formen annehmen. In der Praxis sind die Fragestellungen zur Analyse hierbei jedoch meist recht pragmatisch: So sollte man bspw. beim Design darauf achten, ob man in allen Kanälen eine einheitliche Ansprache benutzt, oder ob das „Dutzen“ und „Siezen“ in nicht nachvollziehbarer Weise wechselt. Daneben ist es natürlich ebenso wichtig, über alle Kanäle hinweg eine Konsistenz in der Angebots- und Preisdarstellung zu bewahren.

\section{Fähigkeiten der Kundlnnen für die Dienstleistung}

Wechselnde Kundenkanäle können auch für KundInnen eine Herausforderung sein. Ihre Beteiligungsqualität in den Leistungserstellungsprozess bei Dienstleistungen hängt von ihren Fähigkeiten ab, das Leistungsangebot des Unternehmens an den unterschiedlichen Kontaktpunkten auch zu nutzen. Es bringt für einen Anbieter wenig, neue Interaktionsmöglichkeiten und Tools anzubieten, die KundInnen schlicht nicht kennen oder sogar überfordern. Insofern ist es erfolgskritisch, an seinen Leistungserstellungsprozess systematisch die Frage zu stellen, welche Fähigkeiten und Ressourcen KundInnen in den einzelnen Schritten benötigen. Zudem muss im Design von Kundenprozessen geklärt werden, welche KundInnen welche Angebote in welchen Kanälen kennen und nutzen. ${ }^{4}$

\section{Qualitätsmonitoring und Weiterentwicklung der gesamten Kundenreise}

Die zunehmende Digitalisierung mit der einhergehenden Vielfalt von miteinander verbundenen Kanälen macht ein permanentes Qualitätsmonitoring der Kundenreisen notwendig. ${ }^{5}$ Ein Qualitätsmonitoring erhebt das Erleben (bspw. Kundenzufriedenheit an einzelnen Kontaktpunkten) und das Verhalten (bspw. Abbrüche beim Onlinekauf, Auflegen bei zu langen Wartezeiten am Telefon). Während für telefonische und persönliche Kontaktpunkte ein entsprechendes Qualitätsmonitoring bereits häufig umgesetzt ist, zeigt sich in der Praxis, dass an neuen Kanä-

"Das Erleben von Kundlnnen in den unterschiedlichen Kanälen muss ein passendes und in sich stimmiges Gesamtbild abgeben." len die Umsetzung oft hinterherhinkt (bspw. bei Chat-Angeboten, Interaktionsmöglichkeiten in Apps oder bei der Videoberatung). Erkenntnisse aus dem Qualitätsmonitoring sind die Basis für eine laufende Weiterentwicklung der Erlebnisqualität einer Kundenreise. Sie sind aber nicht die alleinige Quelle. Erfahrungen und Ideen von Mitarbeitenden und Führungskräften empfehlen sich parallel einzubinden. Dazu gehören auch Erfahrungen aus möglicherweise in der Vergangenheit gescheiterten (Weiter-) Entwicklungsprozessen. 


\section{Gründe für ein Scheitern im Service Design}

Die zusätzlichen Möglichkeiten, die durch die Nutzung analoger und digitalisierter Kundenkanäle entstehen, stellen das Service Design vor neuen Herausforderungen. Es ist alles andere als einfach, Anforderungen von unterschiedlichen Kundengruppen richtig in passende Erlebnisse zu übersetzen, die Übergange zwischen verschiedenen Kanälen reibungslos zu gestalten, die Gesamtreise konsistent zu designen, die Fähigkeiten verschiedener Kundengruppen dabei vor Augen zu haben und die ges. Kundenreise zu monitoren. Insofern ist es wichtig, sich bereits im Entwicklungsprozess klar zu machen, dass die Gestaltung von Dienstleistungsprozessen auch scheitern kann. Es gibt natürlich vielfältige Gründe des Scheiterns. Weit verbreitet sind u.a. folgende: ${ }^{6}$

Scheitern an Wunschdenken: Man scheitert schnell, wenn man Schwierigkeiten und Herausforderungen im Designprozess bagatellisiert. Eine Art „Überoptimismus“ der involvierten Personen begleitet den Designprozess und führt bspw. zur Unterschätzung von Ausgaben für technische Ressourcen um die Übergänge zwischen Kanälen zu gestalten. Oft werden auch die Ausgaben für Marketingmaßnahmen (bspw. Kundentrainings) sowie die benötigte Zeit zur Umsetzung von Designprozessen unterschätzt. Die Abstimmung eines Kundenprozesses über mehrere Kanäle hinweg ist weder günstiger als in einer rein analogen Welt, noch ist das Design der Prozesse schneller.

Scheitern an Selbstüberschätzung: „Overconfidence“ zeigt sich dann, wenn involvierte Personen ihre Fähigkeit, alle Zusammenhänge und Details zu erkennen, überschätzen und in der Folge Fehlentscheidungen treffen. Dies kann sich beispielsweise in einer Überbetonung von eigenen digitalen oder analogen „Lieblingskanälen“ oder der Überschätzung der Kundenfähigkeiten beim Umgang mit digitalen Technologien ausdrücken.

Scheitern am „Not invented here“-Phänomen: Immer wieder findet sich in Teams die Abneigung, Erkenntnisse von anderen aufzunehmen und von bestehenden Ideen, Erfahrungen oder Problemlösungen zu profitieren. Bereits gemachte Fehler im Service Design werden noch einmal gemacht. Auch zu diesem Phänomen zählt es, wenn Ideen von Kunden für die Gestaltung von Services ohne ersichtliche Gründe abgelehnt werden.

Scheitern an „sunk costs": In der Praxis zeigt sich immer wieder, dass an in der Vergangenheit getroffenen Entscheidungen standhaft festgehalten wird, auch wenn klar ist, dass diese falsch waren (bspw. Investitionen in eine IT-Landschaft, welche die benötigte Flexibilität der Kanalübergänge nicht abbilden kann). Hintergrund dieses Verhaltens ist oft, dass man nur ungern einsieht, dass bis dahin bereits investierte Ressourcen einen unwiederbringlichen Verlust darstellen.

\section{Scheitern im Service Design als Failing Forward}

Die oben genannten Gründe des Scheiterns sind nicht nur bei der Entwicklung von Service Designs in einer digitalisieren Welt relevant. Scheitern ist generell ein Tabu in vielen Unternehmen und für viele Führungskräfte. ${ }^{7}$ Konsequenz dieses Tabus ist, dass auf 
individueller wie unternehmerischer Ebene aus den Ursachen des Scheiterns nicht gelernt wird und somit wichtige Impulse für eine Weiterentwicklung des Unternehmens nicht genutzt werden können. Um Lernen zu können, ist es zentral, Ursachen des Scheiterns zu analysieren und die Erfahrungen zu reflektieren und zu kommunizieren. Dies kann helfen, ein zukünftiges Scheitern aus ähnlichen Gründen zu verhindern. Diese eher analytische Auseinandersetzung (,Warum sind wir gescheitert?“) reicht im Umgang mit dem unternehmerischen Scheitern jedoch nicht aus. Damit überhaupt eine konstruktive Auseinandersetzung mit dem unternehmerischen Scheitern möglich ist, benötigt es eine vorwärtsgewandte und neugierige Haltung gegenüber Hintergründen des Scheiterns und eine Motivation diese zu ergründen.

Eine intensive Auseinandersetzung mit dem Scheitern kann einen konstruktiven und vorwärtsgewandten Umgang mit fehlgeschlagenen Kundenreisen ermöglichen. Maxwell (2000) hat dies als „Failing Forward“ bezeichnet. Dieses „Failing Forward" beschreibt im Gegensatz zu einem Failing Backward, aus dem man nichts lernt, einen konstruktiven Umgang mit Fehlern.

Das Failing Forward fordert einen konstruktiven Umgang mit Scheitern im Service Design. Dies ist ein hoher Anspruch. Unterschiedliche Personen können ein Scheitern ganz unterschiedlich bewerten und emotional erleben. Häufig wird das Scheitern um so negativer bewertet, je mehr sich jemand als persönlich gescheitert ansieht. Im Gegensatz dazu wird das Scheitern weniger negativ bewertet, wenn lediglich ein Vorhaben als gescheitert betrachtet wird (siehe Abb. 2). ${ }^{8}$

\begin{tabular}{|l|l|}
\hline Failing Forward & Failing Backward \\
\hline Taking Responsibility & Blaming others \\
\hline Learning from each mistake & Repeating the same mistakes \\
\hline Knowing Failure Is a Part of Progress & Expecting Never to Fail Again \\
\hline Maintaining a Positive Attitude & Expecting to Continually Fail \\
\hline Challenging Outdated Assumptions & Accepting Tradition Blindly \\
\hline Taking New Risks & Being Limited by Past Mistakes \\
\hline Believing Something Didn't Work & Thinking I am a Failure \\
\hline Preserving & Quitting \\
\hline
\end{tabular}

Abbildung 2: Failing Forward vs. Failing Backward9 


\section{Grundvoraussetzungen für ein Failing Forward}

Ein konstruktiver Umgang mit dem Scheitern kann in der Regel nur dann gut erfolgen, wenn das emotionale Erleben des Scheiterns bewusst geworden ist. Starke Emotionen bzw. emotionale Reaktionen wie Ärger und Frustrationen sowie vielleicht Angst vor beruflichen Konsequenzen oder einem Gesichtsverlust durch das Scheitern machen eine vorwärtsgewandte Handhabung des Scheiterns schwer. Sie können häufig zu typischen und wenig konstruktiven Reaktionen führen: nämlich Flucht („Kopf in den Sand“, Verleugnung, etc.) oder Angriff („Fingerpointing“, Suche nach Schuldigen, Vertuschung, etc.). Ein konstruktiver Umgang mit einem Scheitern hängt damit auch stark von der mentalen Stärke einer betroffenen Person ab. Braun et al. (2016) fassen unter dieser mentalen Stärke u.a. Optimismus, Selbstwirksamkeit, Resilienz und Emotionsregulation zusammen. ${ }^{10}$

Unter Optimismus kann eine allgemeine Erwartungshaltung verstanden werden, dass positive Entwicklungen eintreten werden. In Bezug auf Scheitern kann sich ein Optimismus mit Blick sowohl auf das Lernen aus dem Scheitern als auch auf zukünftige Erfolge einstellen. Optimismus verhindert die Entstehung von Hilflosigkeit ${ }^{11}$ und führt dazu, dass negative Erlebnisse weniger "dramatisch“ wahrgenommen werden. Dies gibt Raum für die Reflexion des Scheiterns. Zudem erhalten Optimisten in der Regel mehr soziale Unterstützung und sind anschlussfähiger. Dies ist gerade für den Umgang mit einem Scheitern auf Teamebene besonders wichtig.

Unter der Selbstwirksamkeitserwartung wird die Selbstüberzeugung verstanden, neue oder schwierige Herausforderungen aufgrund der eigenen Kompetenzen zu bewältigen. ${ }^{12}$ Führungskräfte mit hoher Selbstwirksamkeitserwartung gehen davon aus, dass sie es sind, die über den zukünftigen Erfolg (von Projekten etc.) entscheiden. Es liegt an ihnen selber. Fehlt die Selbstwirksamkeitserwartung, wird die Umwelt, die äußeren Umstände für den Erfolg wie für den Misserfolg verantwortlich gemacht. In Situationen des Scheiterns ist die Selbstwirksamkeitserwartung von größter Bedeutung, denn sie umfasst die Überzeugung, mit den Ursachen des Scheiterns erfolgreich umgehen zu können bzw. aus dem Scheitern zu lernen.

Unter Resilienz wird die Fähigkeit verstanden, mit Misserfolgen und belastenden Faktoren gut umzugehen. Resiliente Personen verfügen über die psychische und mentale Widerstandskraft, um erfolgreich mit belastenden Umständen umzugehen und negative Folgen von Stress zu bewältigen. ${ }^{13}$ Je ausgeprägter die individuelle Resilienz, desto vorwärtsgewandter wird der konstruktive Umgang mit einem Scheitern sein.

Emotionsregulation bezeichnet der individuellen Einfluss einer Person auf ihre Emotionen. Dieser Prozess kann automatisch ablaufen oder willentlich gesteuert werden. Zur Emotionsregulation gehört u.a. die Fähigkeit der Aufmerksamkeitslenkung, der kognitiven Umbewertung sowie der Reaktionsmodulation. ${ }^{14}$ Eine individuelle Emotionsregulation ist für eine „Failing Forward“ notwendig. Möglicherweise auftretende negative 
Emotionen wie Ärger, Frustration, Verzweiflung und auch Angst sollten eine konstruktive Verarbeitung des Scheiterns möglichst wenig behindern. Das bewusste Wahrnehmen dieser Emotionen kann helfen, destruktive Verhaltensreaktionen, die automatisch mit einem Scheitern einhergehen, zu verhindern und neue Verhaltensreaktionen zu wählen.

Der Umgang mit einem Scheitern ist nicht nur von individuellen Eigenschaften abhängig. Das Team und die Organisation haben ebenfalls einen nicht zu unterschätzenden Einfluss auf die Verwirklichung eines konstruktiven Failing Forward. Sehr wichtig ist insbesondere, dass man aus dem Scheitern für zukünftige Prozesse im Team lernt. ${ }^{15}$ Die Erfahrungen und Ursachen des Scheiterns müssen reflektiert und konserviert werden. Dies kann beispielsweise auch bedeuten, Kunden in Zukunft noch stärker in die Entwicklung von Kundenreisen einzubinden oder Ergebnisse der internen Arbeit mit Kunden zu verproben. Es ist wichtig, im Team und der Organisation offen über das Scheitern zu sprechen. Wenn dies gelingt, kann die wahrgenommene psychologische Sicherheit in der Organisation und damit ein Erfolgsfaktor für Innovation ausgebaut werden. ${ }^{16}$

Das Design von Serviceprozessen mit vielen unterschiedlichen Kontaktkanälen und Kontaktpunkten ist immer wieder eine Herausforderung für Organisationen. Die Arbeit mit Kundenreisen und Blueprints versucht dieser Komplexität aus Kundensicht greifbar zu begegnen. Es ist wichtig, dass sich Unternehmen um ein bewussten Entwicklungsprozess über alle Kanäle hinweg und eine Kultur des „Failing Forward“ kümmern, da sie wesentliche Erfolgsfaktoren sind, nicht nur in einer digitalisierten Service Welt. Produktiv Scheitern ist untrennbarer Bestandteil des Service Design.

\section{Literatur}

1 Stickdorn, M., \& Schwarzenberger, K. (2016). Service design in tourism. In Siller, H., \& Zehrer, A. (Hrsg.), Entrepreneurship und Tourismus: Unternehmerisches Denken und Erfolgskonzepte aus der Praxis, S. 261-276, 2. Auflage, Wien: Linde Verlag.

2 Shostack, G.L. (1981). How to design a service. In Donnelly, J., \& George, W.R. (Hrsg.). Marketing of Services (S. 221-281). Chicago: American Marketing Association.

3 Daft, R. L., \& Lengel, R. H. (1986). Organizational Information Requirements, Media Richness, and Structural Design. Management Science, 32(5), 554-571.

4 Hamele, T., \& Dathan, O. (2018). Next Generation Client Onboarding. Studie PWC. https://www.pwc.de/en/digitale-transformation/pwc-study-next-generation-client-onboarding-2018.pdf. Zugegriffen am 05.05.2021.

5 Bruhn M. (2020). Qualitätsmanagement für Dienstleistungen. Berlin: Springer.

6 Bedenk, S., \& Mieg, H. A. (2016). Scheitern bei strategischen Entscheidungen. In Kunert, S. (Hrsg.). Failure Management: Ursachen und Folgen des Scheiterns, S. 39-52, Berlin: Springer.

7 Burmeister, L., \& Steinhilper, L. Hrsg. (2015). Gescheiter scheitern. Eine Anleitung für Führungskräfte und Berater. Heidelberg: Carl-Auer.

8 Klüber, K., Carlberg, I. und Bluhm, S. (2015). Scheitern in der individuellen Perspektive. in: Burmeister, L. und Steinhilper, L. (Hrsg.). Gescheiter scheitern. Eine Anleitung für Führungskräfte und Berater, S. 47-52. 2. Auflage, Bern: Carl Auer. 
9 Maxwell, J.C. (2000). Failing forward. Turning mistakes into stepping stones for success. Nashville: Nelson.

10 Braun, O., Gouasé, N., Mihailovic, S., Pfleghar, T., Sauerland, M. (2016). Positive Psychologie und Selbstmanagement: Wege zur mentaler Stärke. Hamburg: Windmühle.

11 Seligman, M. (1991). Pessimisten küsst man nicht. München: Droemer Knaur.

12 Bandura, A. (1979). Sozial-kognitive Lerntheorie. Stuttgart: Klett-Cotta.

13 Braun, O., Gouasé, N., Mihailovic, S., Pfleghar, T., Sauerland, M. (2016). Positive Psychologie und Selbstmanagement: Wege zur mentaler Stärke. Hamburg: Windmühle.

14 Gross, J. J. (1998). The Emerging Field of Emotion Regulation and Mental Health. Clinical Psychology: Science and Practice, (2)3, 151-164.

15 Birkinshaw, J., \& Haas, M. (2016). Fail More Fail Better. London Business School Review, 27(2), 34-37.

16 Edmondson, A.C. (2018). The Fearless Organization: Creating Psychological Safety in the Workplace for Learning, Innovation, and Growth. Hoboken: Wiley.

\section{Information zu den Autoren}

Dr. Andreas Schöler ist Professor für Konsumentenpsychologie und Dienstleistungsmanagement an der Hochschule für angewandtes Management. Zudem ist er Partner der Managementberatung Compagon GmbH \& Co. KG.

Dr. Dominik van Aaken ist Professor für strategisches Management und Organisation an der Universität Salzburg. Zudem ist er Partner der Managementberatung Compagon $\mathrm{GmbH} \&$ Co. KG. 